Groups Geom. Dyn. 4 (2010), 517-532

DOI $10.4171 / \mathrm{GGD} / 93$
Groups, Geometry, and Dynamics

(C) European Mathematical Society

\title{
Isolated points in the space of left orderings of a group
}

\author{
Adam Clay
}

\begin{abstract}
Let $G$ be a left orderable group and $\operatorname{LO}(G)$ the space of all left orderings. We investigate the circumstances under which a left ordering $<$ of $G$ can correspond to an isolated point in $\mathrm{LO}(G)$, in particular we extend the main result of [9] to the case of uncountable groups. With minor technical restrictions on the group $G$, we find that no dense left ordering is isolated in $\operatorname{LO}(G)$, and that the closure of the set of all dense left orderings of $G$ yields a dense $G_{\delta}$ set within a Cantor set of left orderings in $\operatorname{LO}(G)$. Lastly, we show that certain conditions on a discrete left ordering of $G$ can guarantee that it is not isolated in $\operatorname{LO}(G)$, and we illustrate these ideas using the Dehornoy ordering of the braid groups.
\end{abstract}

Mathematics Subject Classification (2010). 06F15, 20F60, 20F36.

Keywords. Ordered groups, Cantor set, isolated points, Dehornoy ordering.

\section{The space of left orderings of a group}

A group $G$ is said to be left-orderable if there exists a strict total ordering $<$ of its elements such that $g<h \Longrightarrow f g<f h$ for all $f, g, h \in G$. Given a left-orderable group $G$ with ordering <, we can identify the left ordering $<$ of $G$ with its positive cone $P=\{g \in G \mid g>1\}$, the set of all positive elements. The positive cone $P$ of a left ordering of a group $G$ satisfies the following two properties:

(1) If $g, h \in P$ then $g h \in P$.

(2) For all $g \in G$, exactly one of $g \in P, g^{-1} \in P$, or $g=1$ holds.

Conversely, given a semigroup $P \subset G$ satisfying the above two properties, we can order the elements of $G$ by specifying that $g<h$ if and only if $g^{-1} h \in P$.

A left ordering $<$ of $G$ is said to be a Conradian ordering if whenever $g, h>1$, then there exists $n \in \mathbb{N}$ such that $g<h g^{n}$. Lastly, a left ordering of a group $G$ is said to be a bi-ordering if the ordering is also invariant under multiplication from the right, namely $g<h \Longrightarrow g f<h f$ for all $f, g, h \in G$. It should be noted that the positive cone $P \subset G$ of a bi-ordering also satisfies the additional property:

(3) For all $g \in G$, we have $g P g^{-1}=P$. 
Analogous to the case of left orderings, a semigroup $P \subset G$ satisfying properties (1)-(3) defines a bi-ordering of $G$.

We can then consider the set $\mathrm{LO}(G) \subset 2^{G}$ of all positive cones in $G$, a space first defined in [12]. As there is a one-to-one correspondence between left orderings of $G$ and positive cones in $G$, it is natural to describe $\operatorname{LO}(G)$ as the space of all left orderings of $G$. The space $\operatorname{LO}(G)$ is endowed with the subspace topology arising from the product topology on $2^{G}$, with a subbasis for the topology on $\operatorname{LO}(G)$ being formed by the open sets $U_{g}=\{P \in \operatorname{LO}(G) \mid g \in P\}$. Note that $\mathrm{LO}(G)$ comes equipped with a natural $G$-action: given an element $g \in G$, the positive cone $P$ is sent by $g$ to its conjugate $g \mathrm{Pg}^{-1}$. Therefore, given a left ordering < of $G$ with positive cone $P$, we can create new left orderings of $G$ by conjugating the corresponding positive cone $P$ by different elements of $G$.

One can check that $\mathrm{LO}(G)$ is a closed subset in $2^{G}$, and from Tychonoff's Theorem we know that $2^{G}$ is compact, so that $\mathrm{LO}(G)$ itself must be a compact space. With this setup, it is also easy to see that $\mathrm{LO}(G)$ is a totally disconnected Hausdorff space, and in [12] it is shown that whenever $G$ is countable, the topology on $\mathrm{LO}(G)$ in fact arises from a very natural metric. Thus we arrive at:

Theorem 1.1 (Sikora, [12]). Let $G$ be a countable group. Then the space $\mathrm{LO}(G)$ is a compact, totally disconnected Hausdorff metric space. If $\mathrm{LO}(G)$ also contains no isolated points, then $\mathrm{LO}(G)$ is homeomorphic to the Cantor set.

Given a group $G$, we would therefore like to address the existence of isolated points in the space $\operatorname{LO}(G)$, as a first step towards understanding the structure of $\mathrm{LO}(G)$.

Recall that a subgroup $C$ of a left-ordered group $G$ is called convex (with respect to the ordering $<$ ) if whenever $f, h \in C$ and $g \in G$, the implication $f<g<h \Longrightarrow$ $g \in C$ holds. For example, it is easy to check that the subgroup $C$ in Proposition 3.1 is convex in the ordering constructed on $G$.

Following [9], we define the Conradian soul $C_{<}(G)$ in a left ordered group $G$ with ordering $<$ to be the largest convex subgroup $C \subset G$ such that the restriction of $<$ to $C$ is a Conradian ordering. Similarly, we use the notation $B_{<}(G)$ to denote the largest convex subgroup $C \subset G$ such that the restriction of $<$ to $C$ is a bi-ordering. Note that we always have $B_{<}(G) \subset C_{<}(G)$, since all bi-orderings are also Conradian orderings.

Using this notation, the main result of [9], which we will extend here to the case of uncountable groups, can be stated as follows.

Theorem 1.2. Let $G$ be a group, and let $P \in \operatorname{LO}(G)$ be an isolated point with associated ordering $<$ of $G$. Then $B_{<}(G)$ is abelian of rank one, and $C_{<}(G)$ is non-trivial and admits only finitely many left orderings.

Note that Theorem 1.2 is proven for the case of countable groups in [9], although the dynamical approach used therein is entirely different from our approach, and does 
not generalize to the case of uncountable groups.

Finally, recall that a left ordering of a group $G$ is dense if whenever $g<h$, then there exists $f \in G$ such that $g<f<h$. If a left ordering $<$ of $G$ is not dense, then it is discrete, meaning that in the ordering $<$ of $G$ there is a least positive element $\epsilon>1$. We explore the structure of $\operatorname{LO}(G)$ by considering the cases of dense and discrete left orderings separately, and we will find:

Theorem 1.3. Let $Z \subset \mathrm{LO}(G)$ denote the set of all dense left orderings of a countable group $G$, and suppose that all rank one abelian subgroups of $G$ are isomorphic to $\mathbb{Z}$. Then if $Z$ is non-empty, its closure $\bar{Z}$ is homeomorphic to the Cantor set, and the set $Z$ is a $G_{\delta}$ set that is dense in $\bar{Z}$.

In the case of abelian groups, our result will be slightly stronger than Theorem 1.3. Specifically, in the case that $G$ is countable and abelian, we will show that $\bar{Z}=$ $\mathrm{LO}(G)$.

Acknowledgments. The author would like to thank Dale Rolfsen, Andrés Navas and Cristóbal Rivas for many useful discussions and comments regarding earlier drafts of this paper.

\section{The case of Conradian orderings}

We first review known results concerning Conradian orderings, and consider also the case of bi-orderings. Note that the results of this section concerning $C_{<}(G)$ appear in [9], and rely on the following difficult lemma ([9] Lemma 4.4), the bulk of which appeared first in [8], and partially in [7].

Lemma 2.1. Suppose that $P$ is the positive cone of a Conradian ordering of the group $G$, and that there is exactly one proper, nontrivial convex subgroup $C \subset G$. Further suppose that both $C$ and $G / C$ are rank one abelian groups. If $P$ is isolated in $\mathrm{LO}(G)$, then $G$ is not bi-orderable.

The next two theorems require the following work of Tararin ([7], Theorem 5.2.1). Recall that a group $G$ admits a finite rational series if

$$
1=G_{0} \triangleleft G_{1} \triangleleft \cdots \triangleleft G_{n}=G
$$

is a finite normal series with all quotients $G_{i+1} / G_{i}$ rank one abelian.

Theorem 2.2. Let $G$ be a left-ordered group.

(1) If $\mathrm{LO}(G)$ is finite, then $G$ has a finite rational series.

(2) Suppose that $G$ has a finite rational series. Then $\operatorname{LO}(G)$ is finite if and only if $G_{i} \triangleleft G$ for all $i$, and none of the quotients $G_{i+2} / G_{i}$ are bi-orderable. Furthermore, in this case the rational series is unique, and for every left ordering of $G$, the convex subgroups are precisely $G_{0}, G_{1}, \ldots, G_{n}$. 
Theorem 2.3 ([9] Proposition 4.1). Suppose that $P$ is the positive cone of a Conradian ordering of $G$. Then $P$ is not an isolated point in the space $\mathrm{LO}(G)$, unless $\mathrm{LO}(G)$ is finite.

Theorem 2.4. Suppose that $P$ is the positive cone of a bi-ordering of $G$. Then $P$ is not isolated in $\mathrm{LO}(G)$ unless $G$ is rank one abelian.

Proof. In the case that $G$ is bi-ordered by the ordering $<$ associated to $P$, we have $C_{<}(G)=G$. From Theorem 2.3, it follows that $G$ itself must have only finitely many left orderings if the bi-ordering $<$ is to have a positive cone that is isolated in $\mathrm{LO}(G)$. However, by the work of Tararin, we see that no group $G$ admitting only finitely many left orders is bi-orderable, except in the case that $G$ is rank one abelian.

\section{Isolated points}

When trying to determine which points in $\mathrm{LO}(G)$ are isolated, the conjugation action on $\mathrm{LO}(G)$ is a useful tool in approximating a given positive cone. Aside from conjugation of a given ordering, there is a second natural way to make new left orderings of $G$, as follows.

Proposition 3.1. Suppose that $C$ is a left-orderable subgroup of $G$ with ordering $\prec$. Suppose also that the left cosets of $C$ can be ordered in a way compatible with group multiplication from the left, namely $a C \prec^{\prime} b C \Longrightarrow c a C \prec^{\prime} c b C$ for all $a, b, c \in G$. Then a left ordering $<$ can be defined on $G$ by specifying a positive cone as follows: An element $g \in G$ satisfies $1<g$ if $g \in C$ and $1 \prec g$, or if $g \notin C$ and $C \prec^{\prime} g C$.

The proof is a simple check. This proposition allows us to change any left ordering of a group $G$ on a specified convex subgroup $C$ : If $C \subset G$ is convex in the left ordering $<$, then convexity allows us to unambiguously define a left-invariant ordering $\prec$ of the cosets $\{g C \mid g \in G\}$. We may then choose a left ordering of $C$ different from $<$, and extend it to a left ordering of $G$ by using the ordering $\prec$ of the cosets, and applying Proposition 3.1.

Next we observe some simple lemmas.

Lemma 3.2. Suppose $P \subset G$ and that $C$ is a convex subgroup of $G$. Then if $P_{C}=P \cap C$ is not an isolated point in $\mathrm{LO}(C), P$ is not an isolated point in $\mathrm{LO}(G)$.

Proof. Suppose that

$$
P \in \bigcap_{i=1}^{m} U_{g_{i}}
$$


and suppose also that we have numbered the elements $g_{i}$ so that $g_{i} \in C$ for $i \leq k$ (possibly $k=0$ in the case that no $g_{i}$ lies in $C$ ). Now in $\operatorname{LO}(C)$, we have that

$$
P_{C} \in \bigcap_{i=1}^{k} U_{g_{i}},
$$

and since $P_{C}$ is not an isolated point, we can choose $P_{C}^{\prime} \in \bigcap_{i=1}^{k} U_{g_{i}}$, with $P_{C}^{\prime} \neq P_{C}$.

We can now construct a positive cone $P^{\prime} \neq P$ on $G$ as follows: Given $g \in G$, $g \in P^{\prime}$ if $g \in C$ and $g \in P_{C}^{\prime}$, or if $g \notin C$ and $g \in P$.

The positive cone $P^{\prime}$ is different from $P$, since $P$ and $P^{\prime}$ disagree on $C$, and by construction, $P^{\prime} \in \bigcap_{i=1}^{m} U_{g_{i}}$. It follows that $P$ is not isolated.

Lemma 3.3. Suppose $P \subset G$ and that $C$ is a normal, convex subgroup of $G$. Let $P^{\prime}$ denote the positive cone of the ordering inherited by the quotient $G / C$. If $P^{\prime}$ is not an isolated point in $\mathrm{LO}(G / C)$, then $P$ is not an isolated point in $\mathrm{LO}(G)$.

The proof is routine.

Lemma 3.4. Let $G$ be a left ordered group with ordering $<$, whose positive cone we denote as $P$. Then the subgroup

$$
\operatorname{stab}(P)=\left\{g \in G \mid g P g^{-1}=P\right\}
$$

is bi-ordered by the restriction of $<$ to $H=\operatorname{stab}(P)$.

Proof. To see that the restriction of $<$ is a bi-ordering, consider its positive cone $P_{H}=P \cap H$. If $g \in P_{H}$ and $h \in H$, then

- $h g h^{-1} \in H$ since $H$ is a subgroup, and

- $h g h^{-1} \in P$ since, by definition, every element of $H$ fixes the positive cone $P$ under conjugation.

Therefore $H$ is bi-ordered.

The main difficulty in characterizing the Conradian soul of an isolated point in $\mathrm{LO}(G)$ is in showing that the Conradian soul is necessarily non-trivial. If $P$ is an isolated point in $\mathrm{LO}(G)$ with associated ordering $<$ of $G$, then $P$ is certainly not an accumulation point of its conjugates in $\operatorname{LO}(G)$. It turns out that knowing $P$ is not an accumulation point of its conjugates $g P g^{-1} \in \mathrm{LO}(G)$ is enough to deduce that $B_{<}(G)$ (and hence $C_{<}(G)$ ) is non-trivial.

Observe that for any group $G$, if $1<h<g$ in the ordering corresponding to $P$, then left multiplication yields $1<h^{-1} g$, and then using the fact that $h$ is positive, we conclude that $1<h^{-1} g h$. Translating this observation into a topological language, we have observed that if $P \in U_{g}$, then $h P h^{-1} \in U_{g}$ for any $h$ with $1<h<g$. Supposing that

$$
\{P\}=\bigcap_{i=1}^{m} U_{g_{i}}
$$


is an isolated point, applying the above trick to the set of elements $\left\{g_{1}, \ldots, g_{n}\right\}$ allows us to conclude that for any $h$ with $1<h<g_{i}$ for all $i \in\{1, \ldots, n\}$, we must have

$$
h P h^{-1} \in \bigcap_{i=1}^{m} U_{g_{i}} .
$$

However, since $P$ is isolated, this means that $h P h^{-1}=P$, so that (in a sense soon to be made more precise) "small elements in $G$ are bi-ordered", as they fix the positive cone $P$ under conjugation.

Lemma 3.5. Suppose that

$$
P \in \bigcap_{i=1}^{m} U_{g_{i}}
$$

where $\left\{g_{1}, \ldots, g_{m}\right\}$ is some finite set of elements of $G$, yet no conjugates of $P$ (different from $P$ itself ) are in this open set. Then there exists $g_{i} \in\left\{g_{1}, \ldots, g_{m}\right\}$ such that the set

$$
C_{i}=\left\{g \in G \mid g_{i}^{-k} \leq g \leq g_{i}^{k} \text { for some } k\right\}
$$

contains only elements of $G$ that fix the positive cone $P$ under conjugation, that is, $g \in C_{i} \Longrightarrow g P g^{-1}=P$.

Proof. First, we show that there exists $g_{i}$ such that all elements in the set

$$
C_{i}^{+}=\left\{g \in G \mid 1<g \leq g_{i}^{k} \text { for some } k\right\}
$$

fix $P$ under conjugation.

To this end, suppose not. Then for each $g_{i}$ there exists $h_{i}$ with $1<h_{i} \leq g_{i}^{k_{i}}$ for some $k_{i}$, and $h_{i} P h_{i}^{-1} \neq P$. Choose $h=\min \left\{h_{1}, \ldots, h_{m}\right\}$. Then for each $i$, we have

$$
h \leq g_{i}^{k_{i}} \Longrightarrow 1 \leq h^{-1} g_{i}^{k_{i}} \Longrightarrow 1<h^{-1} g_{i}^{k_{i}} h,
$$

and therefore $g_{i}^{k_{i}} \in h P h^{-1}$. Now since the element $g_{i}^{k_{i}}$ is positive in the order determined by the positive cone $h P h^{-1}$, its $k_{i}$-th root $g_{i}$ is also positive. This shows that

$$
h P h^{-1} \in \bigcap_{i=1}^{m} U_{g_{i}},
$$

and so $h P h^{-1} \neq P$ by our choice of $h$, a contradiction. Therefore our claim holds for the set $C_{i}^{+}$.

To prove that all elements $g \in C_{i}$ fix the positive cone $P$, suppose that $g \in G$ satisfies $g_{i}^{-k} \leq g<1$ for some $k$. Then $1 \leq g_{i}^{k} g<g_{i}^{k}$, so that either $g=g_{i}^{-k}$ or $g_{i}^{k} g \in C_{i}^{+}$.

(1) In the case $g=g_{i}^{-k}$ we have $g^{-1} \in C_{i}^{+}$and so fixes $P$, and so $g$ fixes $P$ under conjugation. 
(2) If $g_{i}^{k} g \in C_{i}^{+}$we have

$$
g_{i}^{k} g P g^{-1} g_{i}^{-k}=P,
$$

so that we multiply by powers of $g_{i}$ from both sides and find that

$$
g P g^{-1}=g_{i}^{-k} P g_{i}^{k}=P .
$$

Note that case (1) has been used to yield the final equality.

Therefore we have found $g_{i}$ such that all elements in $C_{i}$ fix $P$ as claimed.

Lemma 3.6. For any group $G$, if

$$
P \in \bigcap_{i=1}^{m} U_{g_{i}}
$$

and no conjugates of $P$ distinct from $P$ lie in this open set, then there exists $g_{i}$ such that the set

$$
C_{i}=\left\{g \in G \mid g_{i}^{-k} \leq g \leq g_{i}^{k} \text { for some } k\right\}
$$

is a convex, bi-ordered subgroup of $G$.

Proof. Convexity of $C_{i}$ is clear from the definition. By Lemma 3.5, $C_{i}$ is a subset of the bi-ordered group stab $(P)$, so it follows that $C_{i}$ is bi-ordered by the restriction ordering as well. Being bi-ordered, we can then conclude that $C_{i}$ is a subgroup of $G$ : If $1<g \leq g_{i}^{k}$ for some $k$, then $g_{i}^{-k} \leq g^{-1}<1$, and similarly the implication $a<b$ and $c<d \Longrightarrow a c<b d$ (this implication does not hold for left orders) shows closure under multiplication.

Corollary 3.7. Suppose that the left ordering $<$ of $G$ has positive cone $P$ which is not an accumulation point of its conjugates in $\mathrm{LO}(G)$. Then both $B_{<}(G)$ and $C_{<}(G)$ are non-trivial.

In particular, we have proven that if $<$ corresponds to an isolated point in $\mathrm{LO}(G)$, then both $B_{<}(G)$ and $C_{<}(G)$ are non-trivial.

We are now ready to complete the proof of Theorem 1.2.

Proof of Theorem 1.2. Let $P$ be the positive cone of a left ordering $<$ of a group $G$, and suppose that $P$ is an isolated point in $\operatorname{LO}(G)$. We know that $B_{<}(G)$ and $C_{<}(G)$ are non-trivial by Corollary 3.7, it remains to show that $B_{<}(G)$ is rank one abelian, and that $C_{<}(G)$ admits only finitely many left orderings.

Since we have assumed that $P$ is isolated in $\mathrm{LO}(G)$, it follows from Lemma 3.2 that the restriction of $P$ to $B_{<}(G)$ must define a bi-ordering that is isolated in $\mathrm{LO}\left(B_{<}(G)\right)$. However, by Theorem 2.4, this is only possible in the case when $B_{<}(G)$ is rank one abelian. Similarly, It follows that the restriction of $P$ to $C_{<}(G)$ must define a Conradian ordering that is isolated in $\operatorname{LO}\left(C_{<}(G)\right)$, which by Theorem 2.3 is only possible in the case that $\mathrm{LO}\left(C_{<}(G)\right)$ is finite. 


\section{Dense and discrete orderings}

In recent work ([2], [10]), it has proven fruitful to consider discrete and dense group orderings separately, as they reflect different structures of the underlying group. In considering the structure of $\operatorname{LO}(G)$, dense orderings of a given group $G$ (with minor restrictions on the group $G$ ) are in some sense "generic" in $\operatorname{LO}(G)$, in that dense orderings of $G$ constitute a dense $G_{\delta}$ set inside of a Cantor set within $\operatorname{LO}(G)$. Recall that a set $U$ in a topological space $X$ is a $G_{\delta}$ set if $U$ can be written as a countable intersection of open sets $\left\{U_{i}\right\}_{i=1}^{\infty}$.

Lemma 4.1. Let $Z \subset \mathrm{LO}(G)$ denote the set of dense left orderings of $G$. If $G$ is countable, then $Z$ is a $G_{\delta}$ set.

Proof. Observe that if $\epsilon>1$ is the least positive element in a left ordering $<$ of $G$ with positive cone $P$, then for all $g \in G$ (with $g \neq 1$ different from $\epsilon$ ) either $g<\epsilon^{-1}$ or $\epsilon<g$. In other words, either $P \in U_{g^{-1} \epsilon^{-1}}$ or $P \in U_{\epsilon^{-1} g}$ for all $1 \neq g \in G$ different from $\epsilon$. That is to say, let $V_{\epsilon}$ denote the set of all discrete left orderings of $G$ with least element $\epsilon$. Then we have observed that

$$
V_{\epsilon}=\bigcap_{g \in G \backslash\{1, \epsilon\}}\left(U_{g-1} \epsilon^{-1} \cup U_{\epsilon^{-1} g}\right) \cap U_{\epsilon} .
$$

Note that $V_{\epsilon}$ is closed, as it is an intersection of closed sets, and consists of those positive cones that define an ordering of $G$ with $\epsilon$ as least positive element. Therefore, the set of dense orderings is given by

$$
Z=\bigcap_{1 \neq \epsilon \in G}\left(\operatorname{LO}(G) \backslash V_{\epsilon}\right)
$$

a countable intersection of open sets.

The remaining difficulty is to show that any dense ordering is an accumulation point of other dense orderings. We first consider the case of abelian groups.

4.1. Abelian groups. From [1], we have the following fact:

Proposition 4.2. If $A$ is a torsion-free abelian group with $\operatorname{rank}(A)>1$, then the space $\mathrm{LO}(A)$ has no isolated points.

For a given torsion-free abelian group $A$, we can deduce much more about the structure of $\mathrm{LO}(A)$ by examining the set of all dense orderings of $A$.

Proposition 4.3. Let $P$ be any positive cone in $\mathrm{LO}(A)$, where $A$ is a torsion-free abelian group with $\operatorname{rank}(A)>1$. Then $P$ is an accumulation point of positive cones whose associated orderings are dense orderings. 
We begin by proving a special case.

Lemma 4.4. Let $P$ be any ordering in $\operatorname{LO}\left(\mathbb{Z}^{k}\right)$, where $k>1$. Then $P$ is an accumulation point of dense orderings.

Proof. We follow the ideas of Sikora in [12], making modifications where necessary.

For contradiction, let $k>1$ be the smallest $k$ for which the claim fails. Suppose that

$$
P \in \bigcap_{i=1}^{n} U_{g_{i}},
$$

with no dense orderings in this open set. Note that we may assume that none of the $g_{i}$ 's are integer multiples of one another. Extend the ordering $<$ defined by $P$ to an ordering of $\mathbb{Q}^{k}$ by declaring $v_{1}<v_{2}$ for $v_{1}, v_{2} \in \mathbb{Q}^{k}$ if $n v_{1}<n v_{2}$ whenever $n v_{1}, n v_{2} \in \mathbb{Z}^{k}$. Let $H \subset \mathbb{Q}^{k} \otimes \mathbb{R}=\mathbb{R}^{k}$ be the subset of elements $x \in \mathbb{R}^{k}$ such that every Euclidean neighbourhood of $x$ contains both positive and negative elements. Then $H$ is a hyperplane, and $H$ divides $\mathbb{R}^{k}$ into two components $H_{-}$and $H_{+}$having the property that $H_{+}$contains only positive elements, and $H_{-}$contains only negative elements. Therefore the elements $g_{i}$ lie either in $H_{+}$or $H$ itself.

Suppose that two or more of the elements $\left\{g_{1}, \ldots, g_{n}\right\}$ lie inside $H$. In this case, $H \cap \mathbb{Z}^{k}=\mathbb{Z}^{m}$ for some $m>1$ with $m<k$, and in this case the positive cone $P \cap \mathbb{Z}^{m} \subset \mathbb{Z}^{m}$ cannot be an accumulation point of dense orderings in $\operatorname{LO}\left(\mathbb{Z}^{m}\right)$, for then we could change the positive cone $P$ using Lemma 3.2. This contradicts the minimality of $k$.

The remaining possibilities are that exactly one (or none) of the elements $\left\{g_{1}, \ldots, g_{n}\right\}$ lie inside $H$. In this case, by slight perturbations of the hyperplane $H$, we can produce a new hyperplane $H^{\prime}$ containing none of the lattice points $\mathbb{Z}^{k} \subset \mathbb{R}^{k}$, and with all points $g_{i}$ lying on one side of the hyperplane $H^{\prime}$.

Specifically, suppose that

$$
\boldsymbol{n}=\left(x_{1}, x_{2}, \ldots, x_{k}\right)
$$

is the normal vector defining $H$. Choose real numbers $y_{1}, y_{2}, \ldots, y_{k}$ that are linearly independent over $\mathbb{Q}$, this is possible for every $k>0$ because $\mathbb{R}$ is an abelian group of infinite rank. For each $i=1, \ldots, k$, we may choose a rational number $q_{i}$ such that $q_{i} y_{i}$ is as near to $x_{i}$ as we please, the real numbers $\left\{q_{i} y_{i}\right\}_{i=1}^{k}$ are again linearly independent over $\mathbb{Q}$ and the vector

$$
\boldsymbol{n}^{\prime}=\left(q_{1} y_{1}, q_{2} y_{2}, \ldots, q_{k} y_{k}\right)
$$

can be chosen to be arbitrarily close to $\boldsymbol{n}$. The hyperplane $H^{\prime}$ with normal vector $\boldsymbol{n}^{\prime}$ satisfies $H^{\prime} \cap \mathbb{Z}^{k}=\{0\}$.

If none of the elements $\left\{g_{1}, \ldots, g_{n}\right\}$ lie inside $H$, then we may choose $\boldsymbol{n}^{\prime}$ to be sufficiently close to $\boldsymbol{n}$ so that $\left\{g_{1}, \ldots, g_{n}\right\}$ lie on one side of $H^{\prime}$. If one of the elements $\left\{g_{1}, \ldots, g_{n}\right\}$ lies inside $H$, say $g_{j}$, then we must take more care in choosing 
our normal vector $\boldsymbol{n}^{\prime}$. In this case, we choose $\boldsymbol{n}^{\prime}$ to be close to $\boldsymbol{n}$, and inside of the open set

$$
\left\{x \in \mathbb{R}^{k} \mid \text { the angle between } x \text { and } g_{j} \text { is less than } \frac{\pi}{2}\right\} \text {. }
$$

In either case, this new hyperplane $H^{\prime}$ defines a new ordering $P^{\prime}$ on $\mathbb{Z}^{k}$ by declaring $P^{\prime}=H_{+}^{\prime} \cap \mathbb{Z}^{k}$, where $H_{+}^{\prime}$ is the component of $\mathbb{R}^{k} \backslash H^{\prime}$ containing all $g_{i}$.

To see that this ordering is dense, suppose that $\epsilon \in P^{\prime}$ is the smallest element in $P^{\prime}$. Recall that the normal vector to $H^{\prime}$ is $\boldsymbol{n}^{\prime}$, and therefore the distance between a vector $v$ and the hyperplane $H^{\prime}$ is given by $\left|\operatorname{proj}_{\boldsymbol{n}^{\prime}}(\boldsymbol{v})\right|$, the length of the projection of $\boldsymbol{v}$ onto $\boldsymbol{n}^{\prime}$.

Since $\epsilon$ is the smallest positive element in our ordering, the vector $\epsilon$ must satisfy $\epsilon<v$ for all $v \in P^{\prime}$, which happens if and only if $v-\epsilon \in H_{+}^{\prime}$ for all $v$ in $P^{\prime}$. Hence, for all $v \in P^{\prime}$ we have $\left|\operatorname{proj}_{\boldsymbol{n}^{\prime}}(\boldsymbol{v})\right|>\left|\operatorname{proj}_{\boldsymbol{n}^{\prime}}(\epsilon)\right|$, and so

$$
0<\left|\operatorname{proj}_{\boldsymbol{n}^{\prime}}(\epsilon)\right|=\inf _{\boldsymbol{v} \in P}\left\{\left|\operatorname{proj}_{\boldsymbol{n}^{\prime}}(\boldsymbol{v})\right|\right\} .
$$

For a hyperplane which contains no integer lattice points, this is not possible ([11], Theorem 1.1).

Proof of Proposition 4.3. To prove the statement for an arbitrary torsion-free abelian group $A$ with $\operatorname{rank}(A)>1$, we let $g_{1}, \ldots, g_{m} \in A$ be any finite family of elements in a given positive $P$. We will show that there exist infinitely many positive cones with associated dense orderings on $A$ in which all $g_{i}$ are positive.

Let $N$ be the subgroup of $A$ generated by the elements $g_{1}, \ldots, g_{m}$. Then $N \cong \mathbb{Z}^{k}$ for $k \geq 1$. Assume that $k>1$, for if it is the case that $N \cong \mathbb{Z}$, add an additional generator $g_{m+1}$ none of whose powers lie in $N$-we may do this since $\operatorname{rank}(A)>1$.

By Lemma 4.4, $N$ admits infinitely many dense orderings in which all of $g_{1}, \ldots$, $g_{m}$ are positive, each constructed by perturbations of the hyperplane associated to the restriction order $P_{N}=N \cap P$. Fix a positive cone $P_{N}^{\prime}$ with a dense associated ordering of $N$, with $P_{N}^{\prime} \neq P_{N}$. We may extend $P_{N}^{\prime}$ to a distinct ordering $Q$ on the isolator of $N$

$$
I(N)=\left\{g \in A \mid g^{l} \in N \text { for some } l\right\}
$$

by declaring $g \in Q$ iff $g^{l} \in P_{N}^{\prime}$ for some $l$.

Observe that the ordering of $I(N)$ with positive cone $Q$ is dense, for suppose not, say $Q$ had least element $\epsilon$. Then $\epsilon \in P^{\prime}$ is not possible since $P^{\prime}$ is a dense ordering, so let $l>1$ be the least positive integer such that $\epsilon^{l} \in P^{\prime}$. By density of $P^{\prime}$, we may then choose $g \in P^{\prime} \subset Q$ with $1<g<\epsilon^{l}$. Since the only positive elements less than $\epsilon^{l}$ are $\epsilon, \epsilon^{2}, \ldots, \epsilon^{l-1}$, we have that $g=\epsilon^{i}$ for $i<l$. This contradicts our choice of $l$.

Now $I(N)$ is normal, and the quotient $A / I(N)$ is torsion-free abelian, so we may order the quotient. Using any ordering on the quotient, we can extend the dense ordering of $I(N)$ with positive cone $Q$ to give a dense ordering of $A$ with the required properties. 
Therefore, when $A$ is an abelian group with $\operatorname{rank}(A)>1$, we know that the closure of the set of dense orderings in $\operatorname{LO}(A)$ is the entire space $\operatorname{LO}(A)$. Thus, Proposition 4.3 and Lemma 4.1 together give us the following theorem.

Theorem 4.5. Suppose that $A$ is a countable abelian group. Then $\mathrm{LO}(A)$ is a Cantor set, and the set $Z$ of all dense left orderings of $A$ is a dense $G_{\delta}$ set within $\operatorname{LO}(A)$.

Note that the case of discrete orderings must necessarily be different from this, for there exist abelian groups admitting no discrete orderings: divisible torsion free abelian groups are such an example.

Question 4.6. Let $A$ be a torsion-free abelian group with $\operatorname{rank}(A)>1$. What is the closure of the set of the discrete orderings in $\operatorname{LO}(A)$ ?

4.2. Non-abelian groups. Our results concerning dense orderings generalize to the case of non-abelian groups.

Proposition 4.7. Let $G$ be any group in which all rank one abelian subgroups are isomorphic to $\mathbb{Z}$. If $P \in \mathrm{LO}(G)$ corresponds to a dense left ordering $<$ of $G$, then $P$ is an accumulation point of positive cones whose associated left orderings are dense orderings.

Proof. Let $U=\bigcap_{i=1}^{m} U_{g_{i}}$ be an open set in $\operatorname{LO}(G)$ containing $P$, the positive cone of a dense left ordering < of $G$.

If $U$ contains any conjugates of $P$ (different from $P$ itself), then we are done, so suppose that no conjugate orderings lie in $U$. Then by proposition 3.6, $G$ contains a convex, bi-ordered subgroup $C$ of the form

$$
C=C_{i}=\left\{g \in G: g_{i}^{-k} \leq g \leq g_{i}^{k} \text { for some } k\right\},
$$

where $g_{i} \in\left\{g_{1}, \ldots, g_{m}\right\}$. Denote by $C^{\prime}$ the intersection of all non-trivial convex subgroups of $C$. There are now two cases to consider.

(1) $C^{\prime} \neq\{1\}$. In this case, since $C^{\prime}$ is bi-ordered and contains no convex subgroups, we can use a theorem of Conrad which tells us that the order must be Archimedean, and so $C^{\prime}$ must be abelian. From our assumption on $G$, if $\operatorname{rank}\left(C^{\prime}\right)=$ 1 , we have $C^{\prime} \cong \mathbb{Z}$, meaning that our ordering is discrete. Therefore $\operatorname{rank}\left(C^{\prime}\right)>1$.

Now the restriction ordering on $C^{\prime}$ with positive cone $P \cap C^{\prime}$ is a dense ordering, and we know from Theorem 4.3 that every dense ordering in $\mathrm{LO}\left(C^{\prime}\right)$ is an accumulation point of other dense orderings. Therefore we may change the positive cone $P$ as in the proof of Lemma 3.2, creating a new positive cone $P^{\prime}$ containing all $g_{i}$, and corresponding to a dense ordering of $G$.

(2) $C^{\prime}=\{1\}$. In this case, $C$ must have infinitely many convex subgroups whose intersection is trivial. Therefore, we may choose a convex subgroup $K$ that is nontrivial and contains no $g_{i}$. Define the positive cone of the "flipped ordering" of $K$ to 
be $\left(P^{-1} \cap K\right)=P_{K}^{-1}$. Then we define a new positive cone $P^{\prime} \subset G$, with $P^{\prime} \in U$, by setting $P^{\prime}=P_{K}^{-1} \cup(P \cap G \backslash K)$. Again, the new ordering $<^{\prime}$ of $K$ with positive cone $P^{\prime}$ is dense, and so the ordering we have defined on $G$ is dense.

In the case of an abelian group $A$, the closure of the set of dense orderings was the entire space $\operatorname{LO}(A)$, which is known to be homeomorphic to the Cantor set when $A$ is countable. In the non-abelian case, Theorem 1.3 gives us a similar result.

Proof of Theorem 1.3. Let $G$ be any countable group with all rank one abelian subgroups isomorphic to $\mathbb{Z}$. Then since $G$ is countable, $\operatorname{LO}(G)$ is metrizable, as is the space $\bar{Z} \subset \operatorname{LO}(G)$. Proposition 4.7 shows that the set $\bar{Z}$ contains no isolated points, and since it is closed, it is compact. Therefore $\bar{Z}$ is a compact, metrizable, totally disconnected perfect space, and so is homeomorphic to the Cantor set [6]. By Lemma 4.1, the set $Z$ is also a dense $G_{\delta}$ set within $\bar{Z}$.

With the restriction that all rank one abelian subgroups of $G$ be isomorphic to $\mathbb{Z}$, it also follows readily that any isolated point in $\mathrm{LO}(G)$ must correspond to a discrete left ordering of $G$. This can be seen by appealing to either Theorem 1.3 (which is stronger than what we need), or by appealing to Theorem 1.2, and observing that the smallest convex subgroup in the Conradian soul of an isolated left ordering must be a rank one abelian group.

We turn our attention next to discrete orderings, and observe conditions under which a discrete ordering of $G$ is not an isolated point in $\operatorname{LO}(G)$. We no longer need the restriction that all rank one abelian subgroups be isomorphic to $\mathbb{Z}$.

Lemma 4.8. Suppose that $P \subset G$ is the positive cone of a discrete left ordering $<$ with least element $\epsilon$. Then if $g \epsilon g^{-1}>1$ for all $g \in G$ and

$$
P \in \bigcap_{i=1}^{m} U_{g_{i}}
$$

contains no conjugates of $P$, there exists $g_{i}$ which is not a power of $\epsilon$ such that

$$
C_{i}=\left\{g \in G \mid g_{i}^{-k} \leq g \leq g_{i}^{k} \text { for some } k\right\}
$$

is a convex, bi-ordered subgroup which properly contains the convex subgroup $\langle\epsilon\rangle$.

Proof. Suppose that $U=\bigcap_{i=1}^{m} U_{g_{i}}$ contains $P$, but no conjugates of $P$. If no $g_{i}$ is equal to a power of $\epsilon$, then we are done, as we may apply Proposition 3.6.

On the other hand, suppose that some $g_{i}$ is a power of $\epsilon$, say $g_{1}=\epsilon^{l}$. Then the condition $g \epsilon g^{-1}>1$ for all $g \in G$ guarantees that the open set $U_{\epsilon}$ contains every conjugate of $P$. Therefore, if

$$
\left(\bigcap_{i=2}^{m} U_{g_{i}}\right) \cap U_{\epsilon^{l}}
$$


contains no conjugates of $P$, neither does the open set $\bigcap_{i=2}^{m} U_{g_{i}}$. Continuing to eliminate powers of $\epsilon$ in this way, we can eventually find an open set $\bigcap_{i=r}^{m} U_{g_{i}}$ containing no conjugates of $P$, and with no $g_{i}$ equal to a power of $\epsilon$. From here we may apply Proposition 3.6.

Theorem 4.9. Let $G$ be a group, and $P$ the positive cone of a discrete left ordering $<$ with least positive element $\epsilon$. If $g \in g^{-1} \in P$ for all $g \in G$, then $P$ is not isolated in $\mathrm{LO}(G)$.

Proof. We proceed very similarly to the proof of Theorem 1.3. Let $U=\bigcap_{i=1}^{m} U_{g_{i}}$ be an open set in $\operatorname{LO}(G)$ containing $P$. If $U$ contains any conjugates of $P$, then we are done, so by Lemma 4.8, we may suppose that there exists convex subgroup $C$ properly containing $\langle\epsilon\rangle$, which is bi-ordered by the restriction of $P$.

Note that the convex subgroup $C$ is not rank one abelian: Suppose that $\operatorname{rank}(C)=$ 1. As the containment $\langle\epsilon\rangle \subset C$ is proper, we can choose $c \in C$ with $C \neq 1$, that is not a power of $\epsilon$. If we then assume that $C$ is rank one abelian, we arrive at $\epsilon^{k}=c^{l}$ for some integers $k, l$, contradicting the fact that $\epsilon$ is the least positive element.

Thus, by Theorem 2.4, we know that the restriction of $P$ to the subgroup $C$ is not isolated in $\operatorname{LO}(C)$, and it follows from Lemma 3.2 that $P$ is not isolated in $\operatorname{LO}(G)$.

\section{The braid groups}

As a sample application of these results, we turn our focus to the braid groups. It is known that the space of left orders $\operatorname{LO}\left(B_{n}\right)$ is not homeomorphic to the Cantor set for $n \geq 2$. We begin by defining the Dehornoy left ordering of the braid groups (also known as the 'standard' ordering), whose positive cone we shall denote $P_{\mathrm{D}}$ [4], [3]. Recall that for each integer $n \geq 2$, the Artin braid group $B_{n}$ is the group generated by $\sigma_{1}, \sigma_{2}, \ldots, \sigma_{n-1}$, subject to the relations

$$
\sigma_{i} \sigma_{j}=\sigma_{j} \sigma_{i} \text { if }|i-j|>1, \quad \sigma_{i} \sigma_{j} \sigma_{i}=\sigma_{j} \sigma_{i} \sigma_{j} \text { if }|i-j|=1 .
$$

Definition 5.1. Let $w$ be a word in the generators $\sigma_{i}, \ldots, \sigma_{n-1}$. Then $w$ is said to be $i$-positive if the generator $\sigma_{i}$ occurs in $W$ with only positive exponents, $i$-negative if $\sigma_{i}$ occurs with only negative exponents, and $i$-neutral if $\sigma_{i}$ does not occur in $w$.

It is shown in [3] that for every integer $i$ with $1 \leq i<n$, every braid $\beta \in B_{n}$ is either $i$-positive, $i$-negative, or $i$-neutral. We may then define the positive cone of the Dehornoy ordering:

Definition 5.2. The positive cone $P_{\mathrm{D}} \subset B_{n}$ of the Dehornoy ordering is the set

$$
P_{\mathrm{D}}=\left\{\beta \in B_{n} \mid \beta \text { is } i \text {-positive for some } i \leq n-1\right\} .
$$


There is also a second positive cone of interest, discovered by the authors of [5], which we shall denote by $P_{\mathrm{DD}}$. Denote by $P_{i} \subset B_{n}$ the set of all $i$-positive braids. Note that the set of all $i$-negative braids is simply $P_{i}^{-1}$.

Definition 5.3. The positive cone $P_{\mathrm{DD}} \subset B_{n}$ is the set

$$
P_{\mathrm{DD}}=P_{1} \cup P_{2}^{-1} \cup \cdots \cup P_{n-1}^{(-1)^{n}}
$$

That either of these notions defines a positive cone in $B_{n}$ is difficult to show, as it is not clear that the notion of a braid being $i$-positive is well defined. This was the main idea introduced to braid theorists in Dehornoy's seminal paper [3].

The positive cone $P_{\mathrm{DD}}$ was originally defined in light of the following property:

Proposition 5.4 (Dubrovina, Dubrovin [5]). The positive cone $P_{\mathrm{DD}}$ is generated as a semigroup by the braids

$$
\begin{aligned}
& y_{1}=\sigma_{1} \ldots \sigma_{n-1}, y_{2}=\left(\sigma_{2} \ldots \sigma_{n-1}\right)^{-1}, \\
& y_{3}=\sigma_{3} \ldots \sigma_{n-1}, \ldots, y_{n-1}=\sigma_{n-1}^{(-1)^{n}} .
\end{aligned}
$$

Note that for two positive cones $P$ and $Q$, if $P \subset Q$ then necessarily $P=Q$. Therefore

Corollary 5.5. The order $P_{\mathrm{DD}}$ is an isolated point in $\mathrm{LO}\left(B_{n}\right)$, in particular,

$$
\left\{P_{\mathrm{DD}}\right\}=\bigcap_{i=1}^{n-1} U_{y_{i}} .
$$

Knowing that $\operatorname{LO}\left(B_{n}\right)$ has isolated points for $n \geq 2$, it makes sense to ask the question: Is the standard ordering $P_{\mathrm{D}}$ an isolated point in $\operatorname{LO}\left(B_{n}\right)$ ? This question is answered in [4], using a very explicit calculation. That $P_{\mathrm{D}}$ is not isolated, however, was originally proven in [9], though the techniques are different from those used here, which illustrate our machinery.

First, we begin with a proposition which establishes a very important property of the ordering $P_{\mathrm{D}}$. Recall the Garside monoid $B_{n}^{+} \subset B_{n}$ is the monoid generated by the elements $\sigma_{1}, \ldots, \sigma_{n-1}$.

Proposition 5.6. Let $\beta \in B_{n}$ and $1 \neq \alpha \in B_{n}^{+}$be given. Then $\beta \alpha \beta^{-1} \in P_{\mathrm{D}}$.

This property of the Dehornoy ordering is referred to as the subword property, or property $\mathbf{S}$.

Next we must know that the Dehornoy ordering is discrete [2].

Proposition 5.7. The Dehornoy ordering of $B_{n}$ is discrete, with smallest positive element $\sigma_{n-1}$. 
These two propositions together show us that $P_{\mathrm{D}}$ satisfies the hypotheses of Theorem 4.9. If we can additionally show that $P_{\mathrm{D}}$ has no bi-orderable convex subgroups properly containing $\left\langle\sigma_{n-1}\right\rangle$, then we can conclude that $P_{\mathrm{D}}$ is an accumulation point of its orbit under the $B_{n}$-action on $\operatorname{LO}\left(B_{n}\right)$.

Recall the natural inclusions $B_{m} \subset B_{n}$ whenever $m \leq n$ which takes $\sigma_{i} \in B_{m}$ to $\sigma_{i} \in B_{n}$. A useful operation is the shift homomorphism sh: $B_{m} \rightarrow B_{n}, m<n$ defined by $\operatorname{sh}\left(\sigma_{i}\right)=\sigma_{i+1}$. This is clearly injective and order-preserving. The shift may be iterated, and we note that $\operatorname{sh}^{r}\left(B_{n-r}\right)$ is just the subgroup $\left\langle\sigma_{r+1}, \ldots, \sigma_{n-1}\right\rangle$ of $B_{n}$, or in other words, the subgroup of all elements which are $i$-neutral for all $i \leq r$.

Lemma 5.8. The subgroups $\operatorname{sh}^{r}\left(B_{n-r}\right), r>0$, are the only convex subgroups under the ordering $P_{\mathrm{D}}$.

Proof. Set $H_{r}=\operatorname{sh}^{r}\left(B_{n-r}\right)$, and let $C$ be a convex subgroup in the Dehornoy ordering. Choose $i$ to be the smallest integer such that $C$ contains an $i$-positive braid. Then clearly $C \subset H_{i-1}$. Our aim is to show the opposite inclusion, which establishes the claim.

Let $\beta \in C$ be an $i$-positive braid. The braid $\sigma_{j}^{-1} \beta$ is $i$-positive for $j>i$, so that $1<\sigma_{j}<\beta \Longrightarrow \sigma_{j} \in C$, and so $H_{i} \subset C$. Considering the generator $\sigma_{i}$, we write $\beta=w_{1} \sigma_{i} w_{2}$, where $w_{1}$ is an empty or $i$-neutral word, and $w_{2}$ is an empty, $i$-neutral, or $i$-positive word. We will show $\sigma_{i} \in C$.

First, we note that the braid represented by the word $\sigma_{i} w_{2}$ lies in $C$, as $w_{1}$ contains only $\sigma_{i+1}, \ldots, \sigma_{n-1}$, all of which are in $C$. If $w_{2}$ is empty, the claim is proven, if $w_{2}$ is $i$-neutral, then we may right multiply by appropriate $\sigma_{j}$ for $j>i$ to arrive at $\sigma_{i} \in C$, and again the claim is proven. Lastly, if $w_{2}$ is $i$-positive, then we get

$$
1<w_{2} \Longrightarrow 1<\sigma_{i}<\sigma_{i} w_{2} \in C,
$$

and the claim follows from convexity of $C$.

Since all convex subgroups are isomorphic to a shifted copy of the braid groups, we conclude that

Corollary 5.9. No subgroup that is convex under the ordering $P_{\mathrm{D}}$ is bi-orderable, except for the subgroup $\left\langle\sigma_{n-1}\right\rangle$.

Theorem 5.10. For every $n>2$, the positive cone $P_{\mathrm{D}}$ in $B_{n}$ is an accumulation point of its conjugates in $\mathrm{LO}\left(B_{n}\right)$.

Proof. Apply Corollary 5.9 and Lemma 4.8. 


\section{References}

[1] S. Arworn and Y. Kim, On finitely determined total orders. JP J. Algebra Number Theory Appl. 8 (2007), 177-186. Zbl 1153.06010 MR 2406856

[2] A. Clay and D. Rolfsen, Densely ordered braid subgroups. J. Knot Theory Ramifications 16 (2007), 869-877. Zbl 1139.20033 MR 2354265

[3] P. Dehornoy, Braid groups and left distributive operations. Trans. Amer. Math. Soc. 345 (1994), 115-150. Zbl 0837.20048 MR 1214782

[4] P. Dehornoy, I. Dynnikov, D. Rolfsen, and B. Wiest, Ordering braids. Math. Surveys Monogr. 148, Amer. Math. Soc., Providence, RI, 2008. Zbl 1163.20024 MR 2463428

[5] T. V. Dubrovina and N. I. Dubrovin, On braid groups. Mat. Sb. 192 (2001), 53-64; English transl. Sb. Math. 192 (2001), 693-703. Zbl 1037.20036 MR 1859702

[6] J. G. Hocking and G. S. Young, Topology. Addison-Wesley Publishing Co., Inc., Reading, Mass.-London 1961. Zbl 0135.22701 MR 0125557

[7] V. M. Kopytov and N. Y. Medvedev, Right-ordered groups. Siberian School of Algebra and Logic, Consultants Bureau, New York 1996. Zbl 0852.06005 MR 1393199

[8] P. Linnell, The topology on the space of left orderings of a group. Preprint 2006. arXiv:math/0607470v2 [math.GR]

[9] A. Navas, On the dynamics of (left) orderable groups. Preprint 2007. arXiv:0710.2466v6 [math.GR]

[10] P. A. Linnell, A. H. Rhemtulla, and D. P. O. Rolfsen, Discretely ordered groups. Preprint 2008. arXiv:0808.2686v2 [math.GR]

[11] C. D. Olds, A. Lax, and G. P. Davidoff, The geometry of numbers. Anneli Lax New Math. Libr. 41, Mathematical Association of America, Washington, DC, 2000.

[12] A. S. Sikora, Topology on the spaces of orderings of groups. Bull. London Math. Soc. 36 (2004), 519-526. Zbl 1057.06006 MR 2069015

Received January 7, 2009; revised June 28, 2009

A. Clay, Department of Mathematics, University of British Columbia, Vancouver, B.C., Canada V6T 1 Z2

E-mail: aclay@math.ubc.ca 